Service social

\title{
L'intervention en situation de crise en protection de la jeunesse. Crise familiale ou crise organisationnelle?
}

\section{Christian Dagenais, Camil Bouchard et Julie Turner}

Volume 47, numéro 3-4, 1998

Enfance : enjeux et réalités 2000

URI : https://id.erudit.org/iderudit/706795ar

DOI : https://doi.org/10.7202/706795ar

Aller au sommaire du numéro

\section{Éditeur(s)}

École de service social de l'Université Laval

ISSN

1708-1734 (numérique)

Découvrir la revue

Citer cet article

Dagenais, C., Bouchard, C. \& Turner, J. (1998). L'intervention en situation de crise en protection de la jeunesse. Crise familiale ou crise organisationnelle? Service social, 47(3-4), 41-76. https://doi.org/10.7202/706795ar
Résumé de l'article

Cet article expose les résultats d'une recherche visant à mettre au jour les modèles auxquels se réfèrent implicitement des intervenantes et intervenants de la protection de la jeunesse dans leurs actions en situation de crise. Ces interventions visent des familles signalées en raison des troubles de comportement d'un enfant ou d'un adolescent, d'abus physique ou de négligence. Les résultats permettent de décrire, d'expliquer et de comprendre pourquoi et dans quels contextes les interventions sont mises en oeuvre. Ils révèlent que : a) les intervenants doivent faire face à trois types de crises distincts; b) le stress vécu par certains groupes d'intervenants constitue un élément central de l'intervention en situation de crise, et c) les difficultés liées à la concertation avec les nombreux partenaires du réseau provoquent des insatisfactions majeures chez les intervenants. La discussion porte sur l'impérative nécessité de mieux soutenir les intervenants et de créer une véritable concertation avec les partenaires. 


\title{
L'intervention en situation de crise en protection de la jeunesse Crise familiale ou crise organisationnelle?
}

\author{
Christian DAGENAIS \\ Camil BOUCHARD \\ Julie TURNER
}

Cet article expose les résultats d'une recherche visant à mettre au jour les modèles auxquels se réfèrent implicitement des intervenantes et intervenants de la protection de la jeunesse dans leurs actions en situation de crise. Ces interventions visent des familles signalées en raison des troubles de comportement d'un enfant ou d'un adolescent, d'abus physique ou de négligence. Les résultats permettent de décrire, d'expliquer et de comprendre pourquoi et dans quels contextes les interventions sont mises en œuvre. Ils révèlent que : a) les intervenants doivent faire face à trois types de crises distincts; b) le stress vécu par certains groupes d'intervenants constitue un élément central de l'intervention en situation de crise, et c) les difficultés liées à la concertation avec les nombreux partenaires du réseau provoquent des insatisfactions majeures chez les intervenants. La discussion porte sur l'impérative nécessité de mieux soutenir les intervenants et de créer une véritable concertation avec les partenaires.

The purpose of this paper is to unravel the models which are implicitely referred to by staff members of a Youth Protection Agency when dealing with families in crisis. Families who are reported for child abuse or neglect, and families with youth displaying severe behavior problems, are involved in cases which are selected from staff members' case load. Results identify the reasons and the contextual dimensions driving the workers' interventions in crisis situations. Main findings are to the effect that (a) workers have to deal with three quite distinct types of crises; (b) personal stress is a central feature for specific groups of workers when dealing with families in crisis; (c) problems in collaborating with the service network partners is a major part of the frustration at least in two of the three types of crisis. Results discussion underscores the importance for offering a better support to workers and for installing a much stronger sense of collaboration among the various service partners. 


\section{INTRODUCTION}

Cette recherche vise à mettre au jour les modèles auxquels se réfèrent implicitement les intervenants du Centre de protection de l'enfance et de la jeunesse de Lanaudière (CPEJL) dans leurs interventions auprès de familles en situation de crise. Ces situations impliquent des familles signalées en vertu de la Loi sur la protection de la jeunesse du Québec en raison des troubles de comportement d'un enfant ou d'un adolescent, d'abus physique ou de négligence. Au début de l'année 1993, un comité consultatif, formé d'un groupe d'intervenants et de gestionnaires de l'établissement, duquel émane l'idée de procéder à cette étude, est mandaté pour se joindre aux chercheurs et participer à toutes les étapes de la recherche. Dès la première réunion de ce comité, les membres expriment le souhait d'explorer, de concert avec les chercheurs, l'univers de leur pratique en situation de crise et optent pour une approche de recherche-action participative (RAP) (Reason, 1994; Société royale du Canada, 1995; Stringer, 1996; Tandon, Azelton, Kelly et Strickland, 1998).

La RAP exige la participation active des participantes et des participants tout au long du processus de recherche, depuis la formulation des questions à l'étude à la collecte des données, aux analyses, à l'interprétation des résultats et à leur diffusion (Société royale du Canada, 1995). L'épistémologie de la RAP reconnaît la valeur de la connaissance expérientielle des intervenants (Reason, 1994). En pratique, elle accorde à ceux que les tenants des approches plus traditionnelles ont souvent considérés comme des objets de recherche le droit de parler pour eux-mêmes (Guba et Lincoln, 1989; Huyssens, 1986). Ainsi, dans une RAP, les participants s'engagent activement dans toutes les décisions concernant la recherche. Dans ce contexte, le chercheur ne se présente pas comme un expert, mais plutôt comme une personneressource qui aide les participants à préciser les questions de recherche, contribue à reconnaître la méthode la plus appropriée pour y répondre et encourage les participants à développer leur propre compréhension des résultats (Stringer, 1996). 


\section{Recension des écrits}

Traditionnellement, une recension des écrits vise à synthétiser les connaissances empiriques disponibles sur un sujet donné de façon à cerner les zones à explorer plus en profondeur (Wilson et Hutchison, 1991). Dans une RAP, l'examen de la documentation sert plutôt à s'inspirer des différents modèles et théories de pratique afin d'identifier les caractéristiques, éléments ou dimensions de fond en jeu dans l'intervention en situation de crise. En accord avec notre approche de recherche-action participative, ces dimensions font ensuite l'objet d'une validation auprès des membres du comité consultatif de façon à s'assurer qu'elles reflètent bien la réalité des pratiques et qu'elles permettront d'apporter des éléments de réponse au questionnement à l'origine de cette étude. Les dimensions retenues serviront à orienter les analyses des données recueillies au cours d'entretiens individuels réalisés auprès d'intervenants de l'organisme afin de décrire, de comprendre et d'expliquer ce qui guide leurs actions. Par exemple, dans le cas où le statut socioéconomique de la famille serait retenu en tant que dimension d'analyse, les données seraient examinées de façon à voir comment et en quoi ce statut influence les modèles de pratique des intervenants du CPEJL. Comme nous le verrons dans la section qui suit, la littérature scientifique et professionnelle constitue la première de trois sources d'information sur lesquelles repose cette étude.

\section{Théorie de la crise et modèles d'intervention}

Notre examen de la littérature met en évidence le fait que les modèles d'intervention dont nous disposons actuellement proviennent, pour la plupart, de la théorie de la crise élaborée par Caplan (1964) et Erikson (1959) autour de l'individu en crise. La majorité des modèles d'intervention en situation de crise familiale qui s'inspirent de ces théories remplacent simplement le terme individu par famille (Puryear, 1979; Sugarman et Mashester, 1985, 1986). La plupart des auteurs ne précisent pas qui, dans la famille, est ou n'est pas en crise. Les autres se contentent de mentionner que seulement certains des membres de la famille peuvent être en crise (Bonnefil, 1980; Puryear, 1979). Les programmes d'intervention de crise peuvent viser une vaste gamme d'objectifs et prendre plusieurs formes (Auerbach et 
Stolberg, 1986; Sugarman et Mashester, 1985; Umana, Gross et McConville, 1980). Cependant, dans le contexte de l'intervention de protection de la jeunesse, les modèles les plus étudiés et les plus largement répandus s'inspirent du programme américain Homebuilders. Ce programme s'adresse à des familles dont la crise se définit essentiellement par le risque d'un placement qu'elles souhaitent éviter et que l'intervention vise précisément à écarter (Dagenais, Bégin et Fortin, 1999; Dagenais et Bouchard, 1993, 1996; Kinney, Haapala et Booth, 1991; Nelson, Landsman et Deutelbaum, 1990).

Caplan (1964/1986) décrit la crise comme " une période relativement courte de déséquilibre psychologique chez une personne confrontée à un événement dangereux qui représente un problème pour elle, et qu'elle ne peut fuir ni résoudre avec ses ressources habituelles de solution de problème " (traduit par Lecompte et Lefebvre, 1986, p. 123). Cette définition suppose que la période de turbulence provoquée par l'événement dangereux se résorberait après une période d'environ six semaines. Au plus fort de cette période, la personne en crise serait plus encline à accepter une aide extérieure, car elle aurait épuisé ses ressources pour faire face à la situation; elle se retrouve dans un état d'inconfort et de souffrance qui la rendrait plus vulnérable et ouverte à une aide extérieure (Aguilera, 1990; Du Ranquet, 1991; Golan, 1969; Manning-Kendrick, 1991; Roberts, 1990, 1991). Selon cette conception, de laquelle découlent, rappelons-le, la majorité des modèles d'intervention, la crise constituerait un moment privilégié que certains jugent indispensable au changement (Pittman, 1986; Slive et McConkey-Radetzki, 1989). Cela justifie une intervention rapide et intensive afin de profiter de l'opportunité de changement qu'offre cette courte période. L'ouverture provoquée par la crise déterminerait donc d'une certaine façon le succès de l'intervention (Auerbach et Stolberg, 1986; David, 1987; Gutstein, 1987; Gutstein et collab., 1988; Steele et Raider, 1991).

\section{Contexte de pratique}

De toute évidence, la définition de Caplan (1964) exclut les problèmes à plus long terme, par exemple ceux associés au syndrome de stress post-traumatique, à la détresse accompagnant le décès d'un être cher ou à la " crise d'adolescence » (Fairchild, 
1986; Hendricks et McKean, 1995; Viney, 1976). La plupart des modèles reposent sur la prémisse selon laquelle la famille en crise est ouverte et disposée à recevoir de l'aide. Or, dans un contexte d'intervention familiale, où la sécurité d'un enfant ou d'un adolescent et non pas nécessairement l'équilibre psychologique d'une personne est en cause, la notion de crise elle-même porte à confusion. L'ambiguïté est encore plus grande lorsqu'une famille est amenée à collaborer dans un contexte d'autorité, à la suite d'une dénonciation de la part d'un membre de la famille ou d'une personne extérieure à celle-ci (Everstine et Everstine, 1983; Justice et Justice, 1990). Dans ce contexte précis, la prétention voulant que la crise pourrait faciliter une ouverture à l'aide offerte n'y est plus du tout évidente.

Dans un contexte d'intervention auprès de familles soupçonnées d'abus physique ou de négligence, les intervenants sont fréquemment confrontés à des familles aux prises avec des problèmes chroniques et persistants. Ces familles dites en crise perpétuelle (Berg, 1994; Kagan et Schlosberg, 1989; McCown et Johnson, 1993) manifesteraient peu d'anxiété et les modèles de thérapie familiale basés sur le postulat d'ouverture à l'aide offerte s'avéreraient inefficaces (Coulborn-Faller, 1985; Weitzman, 1985). Dans ces situations, certains soutiennent que seule la menace du retrait du domicile d'un ou des enfants pourrait provoquer une crise et ainsi mettre en place les conditions favorisant la collaboration (Cornille, 1989; Coulshed, 1981; Justice et Justice, 1990; Kagan et Schlosberg, 1989; Minuchin, 1974; O'Hagan, 1984). Mis à part ces quelques modèles coercitifs qui contraignent les familles à recevoir les services et pour lesquels on ne dispose pas de données d'évaluation (Justice et Justice, 1982, 1990; Kagan et Schlosberg, 1989; Manning-Kendrick, 1991), la littérature nous apprend très peu sur la nature des interventions efficaces dans ce type de situation.

\section{Objectif de l'étude}

L'objectif de la présente étude consiste essentiellement à décrire, comprendre et expliquer ce qui guide les actions des intervenants dans des situations dites de crise. Des observations tirées de la pratique montrent que, la plupart du temps, les connaissances des intervenants psychosociaux sont peu formalisées et varient 
énormément d'une personne à l'autre (Finney et Moos, 1987; Patton, 1989). Cela s'explique par le fait que plus nos connaissances augmentent dans un domaine, moins l'on est conscient des détails qui composent ce champ d'expertise, car beaucoup des gestes posés deviennent des automatismes (Fisher, 1990). De fait, il s'avère à peu près impossible d'obtenir, de façon simple, une description cohésive des pratiques lorsque les interventions s'adressent à des familles aux prises avec des problèmes complexes et variés pour lesquels les solutions font rarement l'unanimité. La façon choisie pour y parvenir consiste à travailler avec les principaux intéressés afin de reconstruire explicitement les modèles auxquels ils se réfèrent implicitement dans leur pratique (Patton, 1986, 1990, 1997).

\section{MÉTHOdOLOGIE}

\section{Les participants}

Notre échantillon se compose de 27 intervenants choisis selon deux critères (tableau 1). Le premier critère renvoie au type de fonction qu'ils occupent, soit, d'une part, l'évaluation et l'orientation des familles dont le signalement a été retenu, soit, d'autre part, la prise en charge des familles orientées vers des services à plus long terme à la suite de l'évaluation. Le second critère a trait au type de problématique auquel les intervenants sont confrontés, soit abus physique et négligence ou troubles de comportement. II importe de noter ici que les participants appartiennent à deux catégories professionnelles, soit les agents de relations humaines (équipes 2, 3, 4 et 5) et les éducateurs (équipe 1).

La procédure de sélection des participants consiste à choisir au hasard deux intervenants sur trois dans chacun des cinq sousgroupes afin de constituer un échantillon susceptible de refléter les pratiques en vigueur au sein de chacune des équipes. Chacun des intervenants sélectionnés est invité à un entretien individuel de 60 à 90 minutes où il doit relater trois situations récentes de sa charge de cas. 
Tableau 1

Répartition des participants aux entretiens individuels selon la fonction et la problématique $(n=27)$

\begin{tabular}{lccc}
\hline \multirow{2}{*}{ Problématique } & \multicolumn{3}{c}{ Fonction } \\
\cline { 2 - 4 } & $\begin{array}{c}\text { Éducateurs et } \\
\text { éducatrices }\end{array}$ & $\begin{array}{c}\text { Évaluation I } \\
\text { orientation }\end{array}$ & $\begin{array}{c}\text { Prise en } \\
\text { charge }\end{array}$ \\
\hline $\begin{array}{l}\text { Troubles de } \\
\text { comportement }\end{array}$ & $\begin{array}{c}\text { Équipe 1 } \\
(n=4)\end{array}$ & $\begin{array}{c}\text { Équipe 2 } \\
(n=3)\end{array}$ & $\begin{array}{c}\text { Équipe 3 } \\
(n=7)\end{array}$ \\
$\begin{array}{l}\text { Abus physique } I \\
\text { négligence }\end{array}$ & & $\begin{array}{c}\text { Équipe 4 } \\
(n=5)\end{array}$ & $\begin{array}{c}\text { Équipe 5 } \\
(n=8)\end{array}$ \\
\hline
\end{tabular}

\section{Procédure}

Afin de se préparer à l'entretien, chaque participant est invité à choisir au préalable trois dossiers. Le premier doit porter sur une situation de crise dont le dénouement est satisfaisant à ses yeux et le deuxième, sur une situation de crise dont le dénouement est insatisfaisant. Le troisième dossier doit porter sur une intervention dans une famille où il n'y avait pas de crise. Cette dernière consigne vise à : a) amener les intervenants à prendre le temps de réfléchir, au moment de choisir leurs dossiers, sur ce qui différencie une situation de crise d'une situation de "non-crise » et b) mettre en évidence d'éventuelles différences entre les interventions réalisées dans ces deux contextes. Les portions des entretiens portant sur les situations de "non-crise » servent donc essentiellement de matériel d'ancrage. Les consignes concernant le choix des situations à relater lors des entretiens visent à obtenir un échantillon de situations qui couvrent divers aspects de la pratique. Cet échantillon est constitué de façon à refléter la plupart des points de vue qui existent sur la question (Posavac et Carey, 1992; Wilson et Hutchison, 1991). II ne permet cependant pas de savoir quelle proportion des situations de chaque type les intervenants rencontrent dans leur quotidien, ni d'estimer la quantité de situations de crise auxquelles ils doivent faire face sur une période donnée (Posavac et Carey, 1992; Wilson et Hutchison, 1991). 


\section{Construction de la grille d'analyse des données}

Les dimensions comprises dans la grille d'analyse des données proviennent de trois sources, soit : a) l'examen de la documentation scientifique et professionnelle disponible, b) des séances de discussion tenues auprès des membres du comité consultatif et de différents groupes d'intervenants du CPEJL afin de valider les dimensions extraites de la littérature et d'ajouter les dimensions pertinentes à leurs yeux et c) une opération de cartographie de concepts réalisée auprès d'un autre groupe d'intervenants à une première étape de la recherche.

Au total, douze dimensions sont retenues. Les huit premières proviennent des informations extraites de notre examen de la littérature et des réunions de travail avec les intervenants. La première dimension concerne (1) la définition même de ce qui constitue une crise aux yeux des intervenants. Elle servira à identifier à quel genre de crise les intervenants doivent faire face et leur façon de définir cette crise.

Les deuxième et troisième dimensions retenues ont trait au processus même de l'intervention, soit : (2) la rapidité à intervenir et (3) l'intensité de l'intervention. Compte tenu de la prépondérance accordée à ces deux composantes par la théorie de la crise et par les programmes qui s'en inspirent, ces dimensions feront l'objet d'une attention particulière afin de déterminer dans quelle mesure elles se concrétisent dans les modèles implicites de pratique des intervenants.

Les deux composantes suivantes s'appliquent au domaine des moyens d'intervention. La quatrième dimension correspond (4) au placement. Depuis 1991, à la suite des recommandations du rapport du Groupe de travail sur l'application des mesures de protection de la jeunesse (Boucher et Harvey, 1991), de plus en plus de projets inspirés du modèle Homebuilders ont été mis en place au Québec afin de réduire le recours à la mesure de placement. Les membres du comité consultatif se préoccupent de voir comment les intervenants se servent de cette mesure en situation de crise et comment celle-ci peut contribuer à résoudre les problèmes. La cinquième dimension a trait (5) au développement ou au soutien des pratiques parentales. Elle vise à examiner comment les acti- 
vités appuient le développement des habiletés parentales afin de permettre aux parents de surmonter les difficultés ayant mené au signalement et d'éviter de se retrouver à nouveau dans la même situation.

La sixième dimension concerne (6) l'ouverture des familles à la possibilité de recevoir de l'aide. Elle permettra de voir si, effectivement, certaines familles en crise acceptent volontiers l'aide offerte, de reconnaître les éléments qui peuvent faciliter cette ouverture et de comprendre comment se structurent les interventions lorsque la famille refuse de collaborer.

Les deux dernières dimensions concernent l'intervenant lui-même. La septième a trait (7) au pouvoir légal dont celui-ci dispose. Cette dimension vise à vérifier dans quelle mesure le mandat légal confié aux intervenants de la protection de la jeunesse constitue selon eux un élément facilitant ou un obstacle à la résolution de la crise. Enfin, la huitième a trait (8) aux habiletés professionnelles de l'intervenant; elle vise à déterminer quelles sont, aux yeux des participants, leurs forces et leurs limites en situation de crise. Cette dernière dimension a fait l'objet de nombreuses discussions au sein du comité consultatif et constitue une préoccupation centrale. Une attention particulière sera donc portée à la façon dont les participants perçoivent leurs habiletés professionnelles.

Les quatre autres dimensions résultent d'une opération de cartographie de concepts réalisée avec un groupe de 12 intervenants qui n'ont pas participé aux discussions entourant les dimensions extraites de la littérature. Cette opération fut jugée nécessaire lors des réunions préparatoires à cette étude. Les discussions avaient mis en évidence la confusion entourant certains concepts dans la littérature, notamment en ce qui a trait à la définition de la crise et à la difficulté de cerner les principaux enjeux de l'intervention. II apparaissait dès lors plus approprié de procéder à une opération destinée à identifier ces dimensions préalablement à un examen plus approfondi des pratiques.

La technique de cartographie de concepts (Trochim, 1989a, 1989b; Trochim, Cook et Setze, 1994) a permis de construire avec ce groupe une représentation graphique des principales dimensions en jeu dans l'intervention en situation de crise (Dagenais et Bouchard, 
1995; Dagenais et Bouchard, sous presse). En bref, cette opération met en évidence (9) le stress vécu par les intervenants, (10) les caractéristiques à partir desquelles ils définissent une famille en crise, (11) les limites organisationnelles et (12) les rapports avec les partenaires (tribunal, intervenants de la santé, etc.). Les résultats de cette opération font l'objet d'un second article (Dagenais et Bouchard, sous presse).

\section{Analyse des données}

L'analyse des données, réalisée avec le support informatique du logiciel NUD•IST (Power Version 3.0.5), s'inspire librement de la méthode de comparaison constante, mieux connue sous le nom de grounded theory, mise au point et décrite par Strauss et Corbin $(1990,1997)$. Elle porte sur trois types de traitement des données partiellement juxtaposés. Le premier sert au dépouillement des données, au cours duquel le contenu de la transcription des 1400 pages d'entretiens est décortiqué, phrase par phrase, de façon à donner un nom à toutes les idées, à tous les concepts ou événements en fonction de ce qu'ils représentent ou de ce qui les caractérise. Le deuxième type de traitement, la catégorisation, consiste à regrouper dans une même catégorie les concepts qui font référence à un même phénomène de façon à synthétiser l'ensemble de l'information recueillie lors des entretiens. Chacune des catégories créées fait l'objet d'un consensus entre analystes. Le troisième type de traitement des données, la modélisation, met en relation les résultats obtenus par les regroupements afin de définir les modèles qui s'en dégagent ${ }^{1}$.

\section{RÉSULTATS}

Les résultats sont présentés ici au regard des six thèmes abordés au cours des entretiens, soit : a) le contexte d'intervention; b) la définition de la crise aux yeux des intervenants; c) l'objectif poursuivi pour chacune des interventions; d) les moyens d'intervention mis en œuvre; e) les aspects facilitants et les obstacles aux interventions et f) les éléments de satisfaction et d'insatisfaction.

\footnotetext{
1 Un chapitre de la thèse de doctorat du premier auteur de cet article décrit en détail la procédure d'analyse (Dagenais, en préparation).
} 
Les liens entre ces résultats et les 12 dimensions d'analyse seront explicités dans la section discussion.

\section{Contexte d'intervention}

L'intervention auprès des familles au moment où celles-ci ne sont pas aux prises avec une crise se déroule dans un contexte fort différent des situations de crise. De façon générale, dans les situations sans crise, personne n'est en danger et les membres de la famille reconnaissent le problème; ceux-ci disposent des ressources pour y faire face et collaborent avec l'intervenant. II n'y a ni urgence d'intervenir, ni violence et les acteurs arrivent assez rapidement à une solution pour corriger la situation. II s'agit généralement de situations de crise résorbées depuis un moment ou de dossiers en voie de fermeture.

En situation de crise, les intervenants sont confrontés à une proportion considérable de familles où les crises surviennent de façon cyclique, à des adolescents hors de contrôle, à des situations marquées par la violence, par des problèmes de toxicomanie ou de santé mentale, à des conflits concernant la garde des enfants, à des menaces ou à des tentatives d'homicide sur un enfant et à des parents qui exigent le placement immédiat de leur enfant. L'intervenant doit, en outre, s'accommoder des décisions, des gestes et des pressions d'un grand nombre d'acteurs tels que juges et policiers, des personnes chargées de la réception et du traitement des signalements et d'autres intervenants des centres locaux de services communautaires (CLSC), des centres hospitaliers et de l'école.

\section{Définition de la crise}

Comme le postule la théorie de la crise, dans certaines situations rapportées au cours des entretiens les familles manifestent une ouverture qui permet aux intervenants de mieux comprendre les problèmes. Ceux-ci y voient alors un potentiel de résolution. Mais les intervenants ne considèrent pas toujours la crise comme un événement profitable et celle-ci peut se manifester sur le terrain sous des formes différentes. L'analyse des informations livrées en entretien par les intervenants nous permet, de fait, de distinguer trois types de crises (voir la figure 1). Dans la crise de type 1 (la 
détresse familiale), la famille est aux prises avec un problème qu'elle ne peut résoudre. Les situations de ce type s'apparentent à la définition théorique de la crise : la famille - ou l'un de ses membres - se trouve dans une impasse et a épuisé les ressources dont elle disposait pour faire face à la situation. Ces situations sont généralement marquées par une grande détresse émotionnelle, par la peur de sévices corporels et par l'accumulation de problèmes familiaux ou d'affrontements de plus en plus virulents entre les membres de la famille.

La crise de type 2 (le passage à l'acte) se définit plutôt par son caractère passager ou circonstanciel. Elle se manifeste par des réactions telles que : a) de la colère qui donne lieu à des gestes de violence physique ou verbale de la part d'un membre de la famille; b) une volonté de rompre les liens qui se manifeste par une fugue ou une requête de placement immédiat; ou c) une opposition revêche à l'aide offerte.

La crise de type 3 (la crise organisationnelle) se définit par: a) l'urgence d'intervenir afin de protéger un membre de la famille, généralement un enfant, d'un danger imminent; b) le manque de moyens d'intervention mis à la disposition des professionnels pour faire face à une situation apparemment sans issue; ou c) des atteintes à l'intégrité physique ou à la crédibilité professionnelle de l'intervenant. 


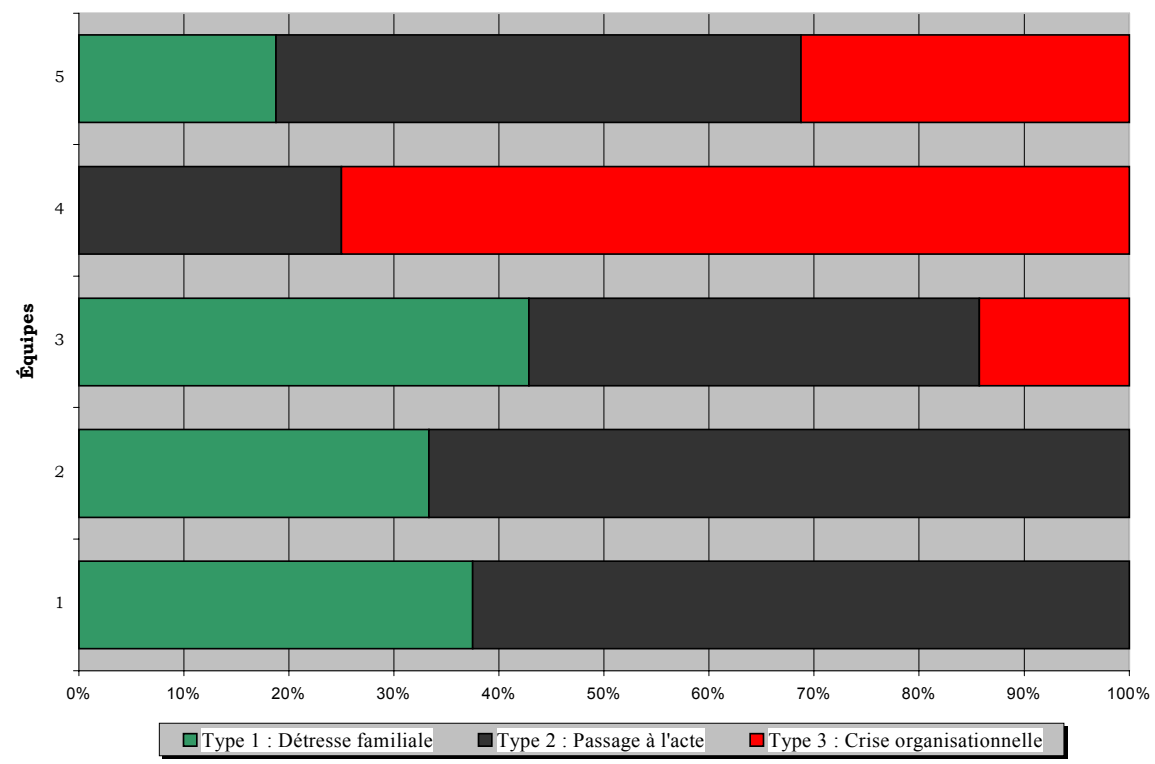

Figure 1 : Type de crise auquel sont confrontés les intervenants de chacune des équipes

Comme le met en évidence la figure 1, les types de crises auxquels les intervenants doivent faire face diffèrent considérablement d'une équipe à l'autre. De fait, les membres des trois équipes qui s'adressent à des familles signalées en troubles de comportement (équipes 1, 2 et 3 ) sont très rarement confrontés à des crises de type 3 . II semble donc que ces intervenants vivent beaucoup moins de stress ou de détresse que les membres des deux équipes en abus physique et négligence (équipes 4 et 5). Ces derniers sont quant à eux rarement appelés à travailler dans le contexte des crises de type 1 où les familles sont plus disposées à recevoir de l'aide. Dans la plupart des cas, ces intervenants doivent composer avec des situations qui se définissent essentiellement par la violence et l'urgence d'intervenir, ce qui les place dans une position de plus grande vulnérabilité.

\section{Objectifs poursuivis par les intervenants}

Nous avons retracé dans le contenu de chacun des entretiens les informations permettant de reconstituer la trajectoire de l'intervention et de définir l'objectif ultime poursuivi. Ainsi que le mettent en 
évidence les figures 2, 3 et 4, l'ensemble de ces objectifs se divise en deux catégories en fonction de la cible principale de l'intervention-l'enfant ou l'adolescent-, représentée par les cellules ombrées, ou en fonction de la dynamique familiale.

Dans les crises de type 1 (détresse familiale), représentées par la figure 2, la famille est aux prises avec un problème grave et se retrouve dans une impasse. Les objectifs visent alors l'amélioration du fonctionnement familial et le maintien ou le retour de l'enfant dans son milieu. Dans certaines situations, l'objectif concerne une seule personne lorsque celle-ci se trouve dans une situation insoutenable et qu'il s'agit d'assurer par exemple un milieu stable à un enfant placé à répétition ou de développer l'autonomie d'une adolescente près de l'âge adulte.

Dans les crises de type 2 (le passage à l'acte), marquées par des épisodes de violence grave, les interventions sont quasi systématiquement centrées sur une seule personne dans la famille : un adolescent ou une adolescente, dans les cas signalés pour troubles de comportement, ou un parent, pour abus physique ou négligence. Par contre, si la situation ne comporte pas d'épisode de violence et que la crise se définit par des réactions d'opposition d'un ou des membres de la famille, des désirs de rupture et des conflits concernant la garde des enfants, l'objectif se centre sur la famille dans son ensemble et vise le maintien dans le milieu, le fonctionnement familial ou la réinsertion familiale (voir la figure 3). 


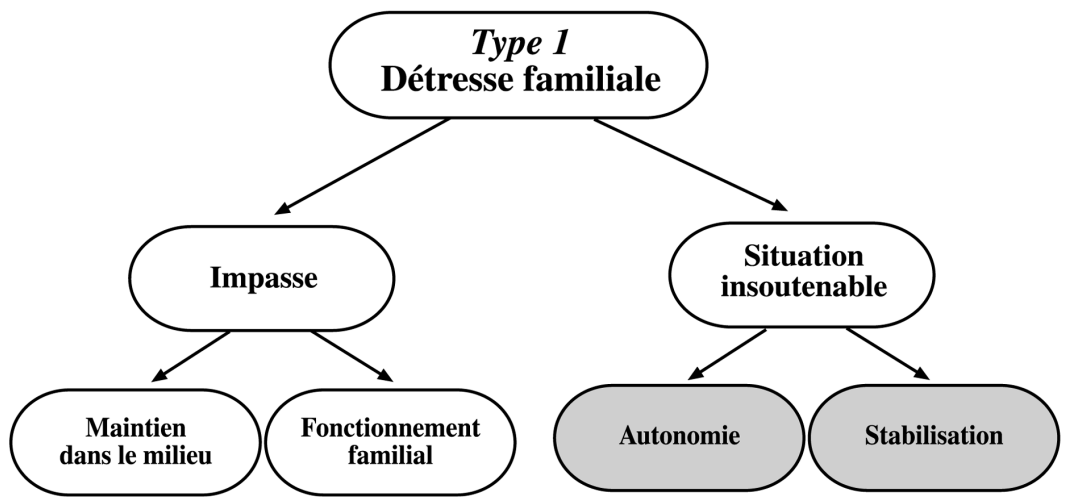

Figure 2 : Objectifs poursuivis dans les crises de type 1

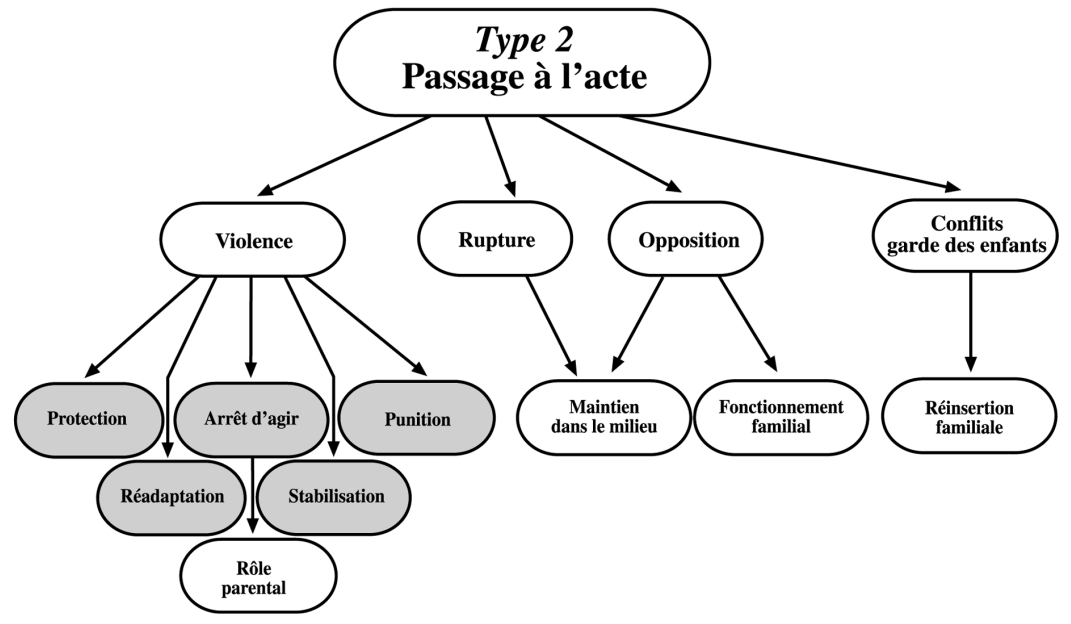

Figure 3 : Objectifs poursuivis dans les crises de type 2

Les crises de type 3 (crise organisationnelle), représentées à la figure 4, se définissent plus souvent par l'urgence d'intervenir face au danger que la situation représente pour un enfant ou un adolescent. L'objectif est alors de protéger et se focalise par le fait même sur un seul membre de la famille. Une fois la sécurité de l'enfant assurée, les intervenants tentent d'agir sur la dynamique familiale. Lorsque les crises de type 3 se définissent par une situation sans issue, par la panique des acteurs ou par une 
atteinte personnelle, l'objectif se centre sur la dynamique familiale et vise le maintien dans le milieu, la réinsertion familiale ou l'amélioration des rôles parentaux.

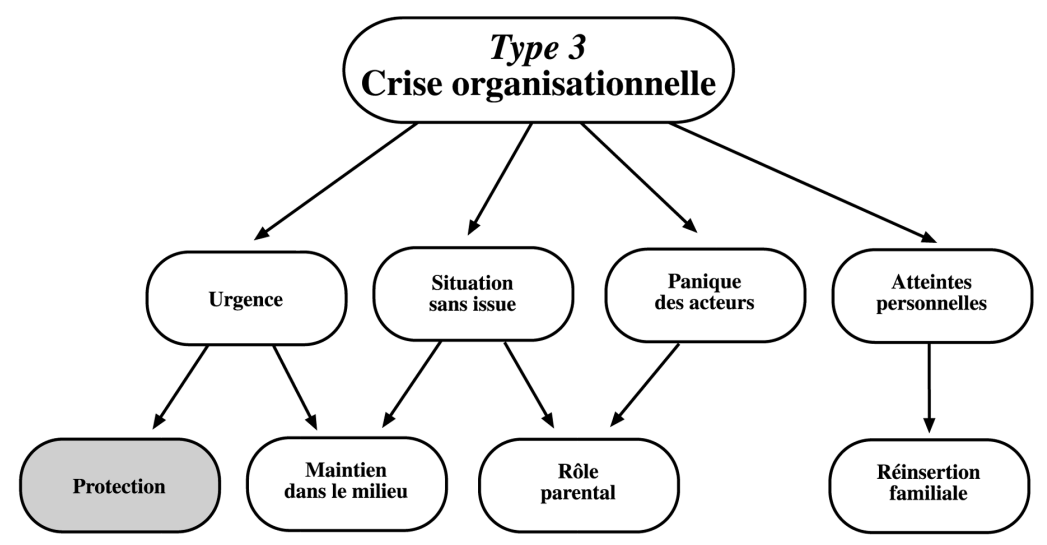

Figure 4 : Objectifs poursuivis dans les crises de type 3

Bref, lorsque la sécurité des enfants n'est pas menacée ou dans les cas où un adolescent n'est pas totalement hors de contrôle, les intervenants orientent leurs actions sur toute la famille plutôt que sur un seul de ses membres. Dans les autres cas, l'intervention visera plutôt la personne vulnérable ou l'agresseur.

\section{Moyens d'intervention}

Les intervenants utilisent une myriade de moyens d'intervention pour aider les familles en crise. Nous les avons répartis sous cinq rubriques, soit : (a) le type de mesure, (b) l'utilisation du placement, (c) le partenariat, (d) l'utilisation de la thérapie et (e) l'intervention en présence d'un ou des membres de la famille.

\section{Type de mesure}

Dans des crises de type 1, la famille manifeste des signes de détresse et semble plus disposée à recevoir de l'aide; cette ouverture se concrétise le plus souvent par la signature de mesures volontaires. Les crises de type 2 impliquent la perte de contrôle d'un ou de plusieurs membres de la famille; les mesures 
judiciaires sont alors très majoritairement utilisées, car, dans ce contexte, peu de gens offrent spontanément leur collaboration. Ces situations impliquent autant des jeunes signalés en troubles de comportement que des familles en abus physique. Dans des crises de type 3 , les mesures volontaires s'avèrent rarement suffisantes pour intervenir et l'on doit recourir au tribunal. Dans ces situations, que l'on rencontre généralement dans les cas de plaintes pour abus physique, des mesures temporaires permettent de protéger les enfants concernés et de clarifier la situation.

\section{Utilisation du placement}

Le placement à court terme constitue un moyen privilégié afin d'assurer la sécurité des enfants ou adolescents, de stopper l'agir des adolescents provisoirement hors de contrôle ou de donner un répit à un parent momentanément inapte à assumer ses responsabilités. Dans bien des cas, le placement ne dure que 24 heures. En situation de crise de type 1, il facilite une atténuation de la tension qui permet d'envisager des solutions appropriées. Le placement à long terme est avant tout utilisé dans le cas d'une problématique persistante ou récurrente : adolescent hors de contrôle, violent et menaçant, ou devant le refus systématique des parents de collaborer de quelque façon que ce soit. II s'agit alors plutôt de crises des types 2 ou 3.

\section{Rapports avec les partenaires}

Lors des placements, les stratégies d'intervention ne semblent pas, du moins pour ce qui entoure la crise elle-même, s'élaborer conjointement avec les intervenants en place. En d'autres mots, lorsqu'un parent d'accueil ou un éducateur est présent, la Direction de la protection de la jeunesse (DPJ) semble moins impliquée, exception faite des situations où, lors du signalement, un lien significatif est déjà établi avec la famille. Les intervenants planifient parfois leurs actions avec le secteur ressource afin de s'assurer de la disponibilité d'une place d'accueil et les avocats du contentieux peuvent aussi être mis à contribution afin de produire des avis légaux. Mais l'équipe à laquelle appartient l'intervenant représente, de loin, sa principale source de soutien. En abus

physique et négligence, il n'est pas rare que, lorsque la situation 
familiale représente un danger potentiel pour l'intervenant (crise de type 3), une intervention en dyade soit réalisée.

La plupart du temps, les rapports avec les intervenants de l'école se limitent à des échanges d'informations. II arrive qu'une collaboration plus étroite s'établisse, mais celle-ci est rarement simple et demande un temps considérable. Dans les situations où un CLSC est impliqué, les intervenants vont échanger des moyens et faire part des stratégies élaborées, surtout dans les cas où un bon contact a été établi avec la famille par l'intervenant ou l'intervenante du CLSC. II arrive assez fréquemment que des intervenants du domaine de la santé soient mis à contribution, mais seulement dans les crises de type 2 et de type 3 signalées pour abus physique ou négligence; il s'agit généralement alors d'obtenir une expertise médicale.

En bref, la collaboration avec les partenaires est le plus souvent limitée à des échanges d'informations. Le fait qu'un autre intervenant (auxiliaire familiale, éducateur ou éducatrice, etc.) déjà au dossier connaisse bien la famille favorise la collaboration; le cas échéant, sa contribution sera mise à profit. Le partenariat semble davantage s'exprimer sous forme de références et de demandes de services que sous forme de concertation ou de mise en commun des compétences de chacun.

\section{Interventions en présence d'un ou des membres de la famille}

Les interventions en face à face avec la famille constituent la dimension pour laquelle les intervenants ont été le plus éloquents. Ces interventions peuvent viser cinq buts différents, soit apaiser, investiguer, impliquer, confronter et transmettre des informations.

Que la crise avec laquelle on doit composer soit chargée d'une grande détresse (type 1) ou qu'elle donne lieu à des gestes de violence (type 2), la première intervention vise habituellement à apaiser les personnes : on tente d'abord et avant tout de ramener le calme. Lorsque la tension baisse suffisamment, des solutions au problème peuvent être envisagées au cours du même entretien.

La deuxième intervention vise, en règle générale, à investiguer. Nous pouvons distinguer trois types d'investigation qui peuvent 
parfois être utilisés successivement par le même intervenant. Le premier consiste à savoir ce qui s'est passé et s'apparente à l'enquête : qu'est-ce qui a déclenché la crise, qui a fait quoi? Le deuxième vise aussi un examen de la situation, mais cette fois afin de comprendre pourquoi cela a pu se passer. Cet examen repose essentiellement sur la définition des besoins de la famille. Le troisième type d'investigation consiste à évaluer les ressources de la famille ou du milieu de vie de l'enfant. L'évaluation des situations signalées pour abus physique présente la particularité qu'on y procède presque invariablement au premier, puis au troisième type d'investigation. On se rappellera que le risque pour l'enfant devient la principale préoccupation des intervenants dans les cas d'abus; dans ces circonstances, on investigue afin de vérifier les faits contenus dans le signalement et d'évaluer le danger potentiel pour les enfants s'ils sont maintenus dans leur milieu.

Dans le deuxième type d'investigation, l'analyse des besoins de la famille est généralement suivie de tentatives d'impliquer ses membres dans des activités visant à répondre à ces besoins. La famille est alors activement mise à contribution afin de trouver des moyens de faire face à la situation qui a provoqué la crise. II est rare que l'intervenant parte d'un a priori et refuse de se laisser influencer par les volontés exprimées. Dans toutes les équipes, lorsque la crise provoque une ouverture, elle permet de clarifier ce que les membres de la famille semblent prêts à faire pour résoudre le problème. Dans la plupart des situations mettant en scène des adolescents, l'intervenant joue le rôle d'intermédiaire entre les besoins et les désirs des parents et du jeune et l'on cherche une solution satisfaisante pour tous. Dans les situations signalées pour abus physique et négligence, l'intervenant pourra tenir compte de la volonté de changement du ou des parents et parfois même revenir sur une décision de placement; il fixera alors des conditions minimales pour assurer la sécurité des enfants concernés.

Dans les situations impliquant des adolescents, on doit parfois utiliser le placement afin d'encadrer un adolescent ou faire pression afin d'en arriver à lui faire prendre conscience de la situation. Les parents pourront également être poussés à assumer leurs responsabilités de parents ou à prendre position face à leur adolescent. Dans d'autres situations, les parents pourront être sensi- 
bilisés à la gravité des gestes posés et aux conséquences que ceux-ci peuvent entraîner pour les enfants.

Une bonne partie du temps des intervenants sert à transmettre des informations aux familles. Ces informations portent sur l'application de la Loi sur la protection de la jeunesse, le rôle de l'intervenant, la possibilité de judiciariser et les étapes du processus judiciaire, les limites du mandat et les conséquences possibles des choix de moyens. La transmission d'informations peut aussi viser à expliquer les comportements d'un adolescent ou porter sur le développement de l'enfant et les conséquences des mauvais traitements.

\section{Aspects facilitants et obstacles à l'intervention}

Nous avons divisé en trois catégories les éléments que les intervenants considèrent comme des facilitants ou des obstacles à l'atteinte des objectifs. II s'agit des caractéristiques (a) des familles, (b) des interventions et (c) de l'organisation.

\section{Caractéristiques des familles}

Les caractéristiques des familles, telles que les ressources personnelles de leurs membres (capacité à se mobiliser ou à demander de l'aide, forte personnalité, intelligence vive, etc.), leur ouverture à recevoir de l'aide et la qualité de leur réseau de soutien, contribuent au succès de l'intervention. Les intervenants de toutes les équipes souhaitent manifestement reconnaître les ressources personnelles des familles et prendre des décisions qui vont dans le sens exprimé par celles-ci. Cela explique sans doute pourquoi l'opposition à l'aide offerte ou le refus de collaborer qui marque beaucoup de situations en abus physique constitue, de loin, le principal obstacle à l'intervention. Cette opposition peut devenir farouche et menaçante, mais souvent elle se manifeste simplement par le refus d'un parent de s'impliquer auprès de son enfant ou de participer à toute forme d'entretien avec l'intervenant.

\section{Caractéristiques des intervenants}

Les attitudes des intervenants, comme la capacité de rester calme devant une désorganisation ou d'exprimer ses limites clairement, 
peuvent aider à atteindre les objectifs. Même si, dans certains cas, ils se reprochent de n'avoir pas consacré suffisamment de temps à entendre ce que la famille avait à dire ou investi autant qu'ils auraient dû, les principales critiques qu'ils s'adressent concernent le contrôle de leurs propres émotions. II en est de même de leurs aptitudes, telles leur expérience professionnelle, leur connaissance des ressources de la région et certaines habiletés particulières comme la facilité d'entrer en relation. Ces obstacles peuvent surgir au sein de toutes les équipes, peu importe le type de crise auquel les intervenants doivent faire face.

\section{Caractéristiques organisationnelles}

La possibilité de recourir à un placement temporaire dans des situations de crise peut amener la famille à prendre conscience de la gravité de la situation; elle permet de "confronter " des jeunes intouchables autrement ou, encore, aide à obtenir des informations nécessaires à la prise de décision. La collaboration des partenaires du réseau de la santé et des services sociaux peut dans certains cas aider à atteindre les objectifs, par exemple lorsque les policiers sont appelés à ramener un jeune fugueur. Cependant, les intervenants peuvent faire une évaluation très négative de la collaboration de leurs partenaires ou se sentir frustrés par les décisions que ceux-ci prennent sans consultation. Encore une fois, ces obstacles peuvent apparaître dans toutes les équipes et ne sont pas associées à un type de crise particulier.

Rappelons que pour la plupart des intervenants la forme de soutien la plus importante vient de l'équipe à laquelle ils appartiennent. Une formation professionnelle non pertinente et la quasiabsence de supervision clinique constitueraient des obstacles à l'intervention. Certains soulignent qu'ils doivent prendre les décisions seuls et, comme ils manquent d'une formation professionnelle adéquate, ils doivent se fier essentiellement à leur intuition pour prendre leurs décisions.

\section{Éléments de satisfaction et d'insatisfaction}

Les intervenants puisent leur principale source de satisfaction dans la possibilité de voir la situation évoluer vers un plus grand bienêtre de la famille et de ses membres et aboutir à une fermeture $d u$ 
dossier. Toutefois, dans les familles présentant des problèmes chroniques, les intervenants ne visent pas nécessairement à fermer le dossier; la seule évolution des familles, même échelonnée sur plusieurs années, peut engendrer une satisfaction considérable. Par contre, l'intervenant peut ressentir une vive insatisfaction à cause de ce qu'il considère comme une incapacité à sortir la famille de l'impasse dans laquelle elle se trouve. II parlera alors d'insatisfactions personnelles, de découragement, de démobilisation et pourra remettre en question ses compétences professionnelles.

Le maintien dans leur milieu des enfants et adolescents constitue une source de satisfaction importante, car plusieurs croient que l'intervention offre plus de chances d'aboutir à un résultat positif pour la famille si celle-ci fait face à la situation sans que l'on ait à procéder à un placement. Dans les situations présentant un danger pour la sécurité des enfants et adolescents, l'intervenant se dira satisfait quand, après un court placement en urgence, l'enfant réintègre son milieu en toute sécurité.

Exception faite des éducateurs et éducatrices, le mandat légal des intervenants constitue selon eux une nécessité, essentiellement parce qu'il permet d'arrêter l'agir d'adolescents hors de contrôle et de protéger les enfants et adolescents. Cependant, l'application de ce mandat peut présenter des inconvénients et les intervenants expriment parfois leur impuissance et leurs frustrations face à un système judiciaire qui, malgré les échecs répétés des tentatives de maintien d'un enfant dans son milieu, persiste à y maintenir ou à y réintégrer les enfants.

En ce qui concerne la collaboration avec les partenaires, bien que les intervenants reconnaissent qu'elle puisse avoir contribué à l'atteinte des objectifs, la qualité de la collaboration avec les partenaires ne semble jamais constituer une source de satisfaction pour les intervenants.

Très souvent, l'insatisfaction provient du fait que les intervenants ne savent pas si les gestes qu'ils ont posés étaient appropriés et s'ils auraient pu faire mieux. Les intervenants œuvrant au sein des équipes en abus physique et négligence doivent gérer le risque que représente l'environnement familial pour l'enfant. Comme on 
pouvait s'y attendre, cette gestion du risque ne se fait pas toujours dans la plus grande sérénité et provoque parfois des inconforts importants. Cette catégorie d'insatisfactions est marquée par le sentiment d'impuissance des intervenants à trouver des solutions adéquates aux problèmes, ce qui les place devant un épuisement de leurs ressources pour faire face à la situation qui peut les amener à se sentir eux-mêmes en crise.

\section{Discussion}

L'examen des données recueillies auprès des intervenants du CPEJL aboutit à trois constats majeurs liés aux douze dimensions d'analyse énumérées au début de cet article. Les sections qui suivent présentent chacun de ces constats ${ }^{2}$.

\section{Trois types de crises}

Le premier constat a trait à l'identification de trois types de crises tels que définis par les intervenants (dimension 1). L'analyse des données montre que l'intervention varie considérablement en fonction de chacun de ces types. Dans des crises de type 1 (détresse familiale), alors que la famille est en demande, les objectifs visent généralement l'amélioration du fonctionnement familial et on tente de maintenir les enfants/adolescents dans leur milieu (dimension 4). Les familles dont il est question dans ce cas présentent rarement des problèmes chroniques et sont mieux disposées à recevoir l'aide offerte (dimension 6). Dans ce cas, les intervenants font appel aux compétences de leurs clients (dimension 10) et tentent (a) de recadrer des parents qui se sentent les seuls responsables des problèmes de leur enfant, (b) d'impliquer les membres des familles dans la recherche de nouveaux moyens de faire face à une situation difficile, (c) de reconnaître des compétences ou des comportements appropriés et $(\mathrm{d})$ de développer de nouvelles habiletés (dimension 5).

2 Même si certaines études arrivent à démontrer un lien entre l'intensité et le succès de l'intervention de crise (Yuan et collab., 1990; Fraser et collab., 1991), il s'avère difficile de déterminer ce que les participants entendent exactement lorsqu'ils parlent d'intervention intensive (dimension 3). Nous avons donc résolu de l'écarter de cette discussion. 
En situation de crise de type 2 (passage à l'acte) et de type 3 (crise organisationnelle), les intervenants assistent très fréquemment à des situations marquées par la violence et ils doivent modifier des dynamiques familiales complexes ou bloquées. Ils sont appelés à atténuer des problèmes cycliques et persistants qu'un grand nombre d'acteurs ne sont pas parvenus à résoudre avant eux. Lorsque la famille n'est pas en demande, comme dans la majorité des crises des types 2 et 3 , il semble que les intervenants ont peu l'occasion de mobiliser les ressources de la famille et de son milieu. Contrairement à ce que plusieurs prétendent (Bloom, 1963; Caplan, 1964; Golan, 1978; Parad et Parad, 1968; Rapoport, 1967; Roberts, 1991), la situation de crise n'offre donc pas de garantie de changement positif à la famille.

Tout porte à croire que les situations de crise des types 2 et 3 , à cause du degré d'urgence qu'elles représentent, exigent une intervention immédiate (dimension 2) et mobilisent la presque totalité des ressources disponibles. Cela laisse peu de place non seulement pour les interventions préventives, mais aussi pour des interventions auprès de familles pour lesquelles le degré d'urgence est peut-être moins élevé. Cela expliquerait pourquoi les énoncés portant sur les limites organisationnelles ont semblé moins importants aux yeux des intervenants lors de l'opération de cartographie de concepts (dimension 11); dans les situations de crise, les praticiens se libèrent rapidement et mobilisent les ressources organisationnelles nécessaires à la résorption du problème.

\section{Stress des intervenants}

Le deuxième constat concerne le stress vécu par les intervenants qui, comme l'a mis en évidence l'opération de cartographie de concepts, s'imposerait à leurs yeux comme la dimension la plus significative de l'intervention en situation de crise (dimension 9). La mise en commun des deux premiers constats laisse supposer que le stress vécu par les intervenants en abus physique et négligence (équipes 4 et 5), souvent aux prises avec des crises des types 2 et 3 , les place dans une position de vulnérabilité importante. Pour la première fois, une définition de l'intervention en situation de crise inclut l'impact de la pratique sur l'intervenant luimême. 
Dans les situations signalées d'abus physique et négligence, on doit souvent recourir à un placement en milieu substitut, parfois parce que le parent exige un retrait immédiat, mais plus souvent parce que l'intervenant considère que la situation met la sécurité de l'enfant en danger et qu'il doit agir de toute urgence (dimension 4). Cet accent sur la protection de l'enfant fait passer au second rang tous les types d'objectifs et constitue une source de stress considérable pour les intervenants de ces équipes. Dans ce contexte, il ne fait aucun doute que le pouvoir légal délégué aux intervenants s'avère indispensable et parfois même vital (dimension 7).

Une autre importante source de stress pour les intervenants de ces deux équipes vient du fait que leur propre sécurité semble menacée régulièrement par leurs clients dans les actions qu'ils doivent réaliser lors des situations de crise. Ces menaces sont généralement liées à la possibilité de retirer les enfants de leur milieu. Répétons-le : la préoccupation d'assurer leur propre sécurité fait partie du quotidien des intervenants. Les intervenants des trois équipes en troubles de comportement (équipes 1, 2 et 3), qui s'adressent à des familles aux prises avec un adolescent en difficulté, semblent soumis à un moins grand stress. Dans ces cas, souvent des crises de type 1 , les parents manifestent une grande ouverture à la possibilité de recevoir de l'aide.

\section{Partenariat}

Le troisième constat met en évidence les difficultés liées à la concertation avec les nombreux partenaires. Lorsqu'ils sont interrogés sur leurs sources de satisfaction et d'insatisfaction, les intervenants mentionnent fréquemment que la collaboration des partenaires provoque des frustrations et des insatisfactions, mais jamais de satisfaction. Ces résultats mettent en évidence les difficultés associées au travail en concertation (dimension 12). La question qui se pose alors concerne la meilleure façon de mettre en commun les expertises locales et régionales au profit des familles: à l'intérieur même des équipes, entre les équipes et avec les partenaires. II y a nettement là matière à innovation nécessaire au bien-être des intervenants, à celui des familles et des enfants et à l'efficience de l'organisation (Gouvernement du Québec, 1998; Schorr et Schorr, 1988). 
Les intervenants participant à cette étude sont maintenant répartis en fonction du territoire plutôt que par problématique, ce qui semble à première vue positif. Cela leur permet de mieux connaître les ressources de leur milieu respectif. Cette nouvelle répartition favorise sûrement une: « ... meilleure connaissance des ressources... une analyse plus articulée des conditions objectives de vie, un contact plus facile entre intervenants et famille en dehors du contexte "problème", une meilleure articulation des efforts avec ceux d'autres ressources formelles ou semi-formelles » (Bouchard, 1987, p. 471).

Cependant, rappelons que nos résultats montrent que l'intervenant trouve sa principale source de soutien au sein de l'équipe à laquelle il appartient. Les regroupements par territoire ont provoqué un effritement des équipes auparavant regroupées par problématique. Parce que les intervenants travaillaient autour de la même problématique depuis plusieurs années, ces équipes offraient cohésion et soutien. Théoriquement, les changements récents pourraient bien favoriser une meilleure concertation des partenaires, mais ils accentuent du même coup l'isolement des intervenants dans leur propre organisation si celle-ci n'adopte pas une configuration conséquente. Cela les prive de leur principale source de soutien et les place dans une position de vulnérabilité importante (Brown et O'Brien, 1998; Davies, 1989).

De fait, à la suite des nombreuses compressions budgétaires et de l'augmentation du nombre d'enfants signalés aux organismes de protection, les stratégies se focalisent généralement sur des aménagements au niveau des structures de services (Gouvernement du Québec, 1998). Ces innovations qui ont brutalement secoué les centres jeunesse du Québec au cours des vagues successives de réorganisation des dernières années reposent sur deux convictions: (a) une meilleure coordination des services garantira aux enfants l'intervention la plus adéquate et (b) le service le plus approprié mènera nécessairement à un meilleur résultat pour l'enfant. Malheureusement, à ce jour, absolument aucune recherche empirique ne confirme ces convictions apparemment logiques (Hoagwood, 1997) et certaines études montrent même un effet négatif (Glissen et Hemmelgarn, 1998). 


\section{CONCLUSION}

Dans un contexte marqué par le stress, la violence et la gravité des situations auxquelles ils doivent faire face, nous avons vu que les intervenants semblent trouver du soutien presque exclusivement auprès de leurs collègues. Sauf lorsque son supérieur immédiat avait appuyé les actions déjà envisagées par l'intervenant au dossier, personne n'a mentionné que le soutien offert par celui-ci avait contribué à résoudre la situation. De fait, le soutien professionnel offert aux intervenants est pour ainsi dire passé inaperçu lors de l'examen des 81 situations d'intervention soumises en entretien individuel. De même, aucun intervenant n'a rapporté que la formation qu'il avait reçue avait contribué à résoudre les problèmes de la famille.

Rappelons que la présente étude découle de l'initiative d'un groupe d'intervenants et de gestionnaires du CPEJL. L'authentique dialogue qui s'est installé entre ces personnes et les chercheurs, regroupés en un comité consultatif, s'est poursuivi tout au long du processus, des activités de recherche et de l'interprétation des résultats. Mentionnons qu'une version préliminaire du rapport a fait l'objet d'un examen attentif des membres de ce comité. Cette version de plus de 180 pages du rapport a fait l'objet d'une lecture à haute voix, phrase par phrase, lors d'une série de cinq demi-journées de réunion du comité consultatif. Ce travail, à première vue rebutant et fastidieux, s'est révélé captivant et fécond, autant du point de vue des chercheurs que pour l'ensemble des membres du comité. De fait, cette opération a permis : 1) d'éliminer du rapport final toute expression ne faisant pas partie de la culture de l'établissement; 2) de définir, par consensus, les enjeux prioritaires liés aux résultats mis en lumière par nos travaux; 3) d'anticiper l'accueil que pourraient recevoir ces résultats de la part des intervenants et de la direction de l'établissement; 4) de tracer les grandes lignes du contenu de la discussion; 5) d'opter pour un format de présentation du rapport d'un maximum de 50 pages et 6 ) de choisir de repousser vers des appendices les informations destinées à rendre compte de la méthode employée, de la procédure d'analyse des données et de la rigueur de l'ensemble de la démarche. La seule contrainte imposée par le chercheur concerne la transparence de la démarche, 
aucun des constats découlant de l'analyse ne pouvant faire l'objet d'une censure.

Selon les tenants de ce type d'approche, la RAP aiderait à : « rendre les questions de recherche plus pertinentes, les méthodes acceptables et les résultats plus significatifs pour la population qui en fait l'objet » (Société royale du Canada, 1995, p. vii). Selon Pieper (1985), elle permettrait également d'éviter les erreurs de type $\mathrm{III}^{3}$, qui consistent à apporter des solutions au mauvais problème. La RAP améliorerait donc non seulement la validité des conclusions (Daughtry et Kundel, 1993), mais aussi les probabilités que celles-ci soient utilisées (Linton, 1989; Wiener et collab., 1991).

Notre expérience montre que, de fait, peu de temps après la réunion de présentation des résultats en octobre 1997, un comité de réflexion sur la formation, le perfectionnement, le soutien et l'encadrement professionnel au CPEJL fut mis sur pied. À la suite des discussion sur ce que ce comité qualifie de "besoins criants d'un plan d'action pour le développement du personnel », il recommande l'instauration d'une véritable dynamique de développement professionnel. En juillet 1998, un nouveau sous-comité, soutenu par un consultant externe, est formé afin d'entreprendre une démarche concrète de développement et de soutien du personnel. Le mandat de consultation, confié à un nouveau groupe de chercheurs, consistait à soutenir le comité dans :

la réalisation d'un diagnostic sur les besoins actuels et concrets du personnel des $\mathrm{CJ}$ de Lanaudière en matière de développement de leurs connaissances et habiletés ainsi qu'à proposer un plan d'action quant aux moyens de soutien organisationnel susceptibles d'améliorer leur situation et le climat général dans l'organisation du travail (Larivière et Barbeau, 1999).

Bien sûr, cet aboutissement heureux ne peut être attribué au seul choix d'une approche de recherche-action participative. II faut en effet considérer l'impact de la réforme des services sociaux, qui a

3 Les erreurs de type I et de type II consistent respectivement: 1) à détecter un effet alors qu'il n'y en a pas et 2) à ne pas détecter un effet alors qu'il y en a un. 
secoué l'ensemble des centres jeunesse du Québec, bien avant que nos conclusions soient présentées. De même, à peine quelques mois après le dévoilement de nos résultats, la Commission des droits de la personne et des droits de la jeunesse déposait son rapport à la suite de la triste affaire du " bourreau de Beaumont ». Ces circonstances ont sans aucun doute favorisé la mobilisation de l'ensemble des directions d'établissements de protection de la jeunesse au Québec autour de la question de l'encadrement de leur personnel. Au CPEJ de Lanaudière, les résultats produits par notre étude ont favorisé une réflexion sur la meilleure façon de répondre aux besoins exprimés par ceux-là mêmes qui interviennent auprès des enfants et de leur famille, plutôt que de se centrer sur des mesures de contrôle.

Rappelons que cette étude ne visait pas à porter un jugement sur la pertinence ou la qualité des interventions réalisées, mais plutôt à décrire, à expliquer et à comprendre les éléments qui incitent les intervenants à poser tel ou tel geste. II s'agissait donc de mettre au jour, ou de découvrir, les modèles qui guident les intervenants à partir de l'interprétation de leur discours (Tandon et collab. 1998). Dans ce contexte, plutôt que de procéder à un traitement statistique quantitatif, les modèles sont élaborés de façon à rendre compte de l'ensemble des différentes décisions possibles, des causes et des conséquences de ces décisions et des variables qui les influencent (Wilson et Hutchison, 1991). De cette façon, les modèles élaborés sont plus susceptibles de refléter la réalité des principaux intéressés, d'être intelligibles, perfectibles et utilisables. (Turner, 1983). Les décisions prises au cours de l'année qui a suivi le dépôt de notre rapport au CPEJL fortifient cette conviction.

Selon les tenants des approches quantitatives traditionnelles, la validité se mesure par le degré de concordance entre deux mesures du même trait à l'aide de méthodes différentes (Campbell et Fiske, 1959). La confiance à l'égard des résultats produits par des approches qualitatives ne peut quant à elle s'évaluer qu'en fonction de leur vraisemblance (Polkinghorne, 1988; Miles et Huberman, 1994). Dans le cas qui nous occupe, le travail de concert avec un comité consultatif, considéré comme un groupe d'experts, confère à nos résultats cette vraisemblance. L'accueil réservé à nos conclusions par l'ensemble du personnel de l'établissement leur attribue une crédibilité encore plus grande (Fawcett, 1990). 
II convient cependant de rappeler que cette étude ne porte que sur la seule expérience des intervenantes et intervenants de cinq équipes du CPEJ de Lanaudière. Les conclusions qui en découlent ne peuvent donc être généralisées à d'autres établissements qu'avec prudence. Cela est encore plus vrai lorsque l'on considère les nombreux changements survenus dans les services aux jeunes et à leur famille depuis le moment où nous avons recueilli nos données. Mentionnons toutefois que les résultats de cette étude ont fait l'objet d'une vaste opération de diffusion et de réflexion dans les établissements de services aux enfants et à leur famille. Plus d'une dizaine de séminaires ont été présentés dans la périphérie de Montréal, à la table des directeurs des services professionnels ainsi qu'à la table des directeurs généraux de l'ensemble des centres jeunesse du Québec. Aux yeux de tous, ces résultats ont fait du sens. Cela nous porte à croire qu'ils portent toujours de précieuses indications pour les établissements de services aux enfants et à leur famille.

Christian DAGENAIS

Chercheur associé

Groupe de recherche et d'action sur la victimisation des enfants (GRAVE)

Camil BOUCHARD

Professeur Département de psychologie Université du Québec à Montréal

Julie TURNER Assistante de recherche Laboratoire de recherche en écologie humaine et sociale (LAREHS) Université du Québec à Montréal 


\section{Références bibliographiques}

AgUILERA, D.C. (1990). Crisis Intervention: Theory and Methodology, St. Louis, MO, C.V. Mosby.

Auerbach, S.M. et A.L. Stolberg (1986). Crisis intervention with children and families, Washington, DC, Hemisphere.

BONNEFIL, M.C. (1979). "Therapist: Save my child: A family crisis case », Clinical Social Work Journal, vol. 7, p. 6-14.

BoUCHARD, C. (1987). "Intervenir à partir de l'approche écologique: au centre, l'intervenante ", Service social, vol. 36, nos 2 et 3, p. 454-477.

BOUCHER, L. et J. HARVEY (1991). La protection sur mesure: Un projet collectif. Rapport du groupe de travail sur l'application des mesures de protection de la jeunesse, Québec, ministère de la Santé et des Services sociaux.

BROWN, C. et K.M. O'BRIEN (1998). "Understanding stress and burnout in shelter workers », Professional-Psychology: Research-and-Practice, vol. 29, $\mathrm{n}^{\circ} 4$, p. 383-385.

CAMPBELL, D.T. et D.W. FISKE (1959). "Convergent and discriminant validation by the multitrait-multimethod matrix ", Psychological Bulletin, vol. 56, p. 81-105.

CAPLAN, G. (1964). Principles of preventive psychiatry, New York, Basic Books.

CORNILLE, T.A. (1989). « Family therapy and social control with incestuous families », Contemporary Family Therapy, vol. 11, p. 101-117.

COULBORN FALLER, K. (1985). " Unanticipated problems in the United States child protection system », Child Abuse and Neglect, vol. 9, p. 63-69.

COULSHED, V. (1981). «Engaging in family therapy: problems for the inexperimented uninvited therapist », Journal of Family Therapy, vol. 3, p. 27-38.

DAGENAIS, C., J. BÉGIN et D. ForTIN (1999). L'évaluation des services intensifs offerts à des familles en crise et l'amélioration du fonctionnement familial. Une méta-analyse. Les cahiers d'analyse du GRAVE, vol. 6, no 1. Montréal, Université du Québec à Montréal, Laboratoire de recherche en écologie humaine et sociale. 
DAGENAIS, C. et C. BouChARD (1993). «Les programmes de soutien intensif aux familles. Intervention massive ou intervention magique? », P.R.I.S.M.E., vol. 3, p. 503-515.

DAGENAIS, C. et C. BOUCHARD (1995). La cartographie de concepts dans le contexte de la modélisation des interventions auprès de familles en crise. Les cahiers d'analyse du GRAVE, vol. 2, n० 3 , Montréal, Université du Québec à Montréal, Laboratoire de recherche en écologie humaine et sociale.

DAGENAIS, C. et C. BouChARD (1996). "L'impact des programmes de soutien intensif visant à maintenir les enfants et adolescents dans leur famille », Revue canadienne de santé mentale communautaire, vol. $15, \mathrm{n}^{\circ} 1$, p. 63-82.

DAGENAIS, C. et C. BOUCHARD (sous presse). "Contribution de la cartographie de concepts à la modélisation des interventions en situation de crise en protection de la jeunesse ». Article soumis pour publication à la Revue canadienne d'évaluation de programme.

DAUGHTRY, D. et M.A. KUNDEL (1993). «Experience of depression in college students: A concept map », Journal of Counseling Psychology, vol. 40, n 3, p. 316-323.

DAVID, G. (1987). " Nécessité et efficacité d'une approche intégrée dans le traitement de l'inceste ", Service social, vol. 36, p. 350-368.

DAVIES, L. (1989). «Professional autonomy revisited: The case of British social work practice in child abuse », Canadian Social Work Review, vol. 6, p. 186-202.

DU RANQUET, M. (1991). Les approches en service social, Québec, Edisem.

ERIKSON, E.H. (1959). "Identity and the life cycle ", Psychological Issues, 1, p. 1-171.

Everstine, D.S. et L. Everstine (1983). People in crisis: Strategic Interventions, New York, Brunner-Mazel.

FAIRCHILD, T.N. (1986). Crisis intervention strategies for school-based helpers, Springfield, IL, Charles C. Thomas.

FAWCETT, S. (1990). " Some emerging standards for community research and actions: Aid from a behavioral perspective », dans P. Tolan, C. Keys, F. Chertok et L. Jackson (dir.), Researching community psychology (p. 64-75), Washington, DC, American Psychological Association.

FINNEY, J.W. et R.H. Moos (1987). «Environmental assessment and evaluation research: examples from mental health and substance 
abuse programs », Evaluation Studies Review Annual, vol. 11, p. $564-579$.

FISHER, K.M. (1990). "Semantic Networking: The new kid on the block », Journal of research in science teaching, vol. 27, p. 1001-1018.

Glisson, C. et A. Hemmelgarn (1998). «The effect of organizational climate and interorganizational coordination on the quality and outcomes of children's service system ", Child Abuse and Neglect, vol. 22, n' 5, p. 401-421.

GolAN, N. (1969). "When is a client in crisis? » Social Casework, juillet, p. 389-394.

GouVERnEMENT DU QUÉBEC (1998). Pour une stratégie de soutien du développement des enfants et des jeunes : Agissons en complices, Québec, ministère de la Santé et des Services sociaux.

GuBA, E.G. et Y.S. LINCOLN (1989). Fourth Generation Evaluation, Newbury Park, NY, Sage.

Gutstein, S.E., F.M. RUDd, C.J. GRAHAM et L.L. RAyHA (1988). "Systemic crisis intervention as a response to adolescent crisis: An outcome study », Family Process, 27, p. 201-211.

HENDRICKS, J.E. et J.B. MCKeAN (1995). Crisis intervention: Contemporary issues for on-site interveners ( $2^{\mathrm{e}}$ éd.), Springfield, IL, Charles C. Thomas.

HOAGWOOD, K. (1997). « Intrepreting nullity: The Fort Bragg experiment a comparative success or failure? " American Psychologist, vol. 52, p. 546-550.

HUYSSENS, A. (1986). After the great dividee: Modernism, mass culture, postmodernism, Bloomington, IN, Indiana University Press.

JUSTICE, B. et R. JUSTICE (1982). «Etiology fo physical abuse of children and dynamics of coercitive treatment ", dans J.C. Hansen et L.R. Barnhill (dir.), Clinical approaches to family violence, Rockville, MD, Aspen.

Justice, R. et B. JUSTICE (1990). "Crisis intervention with abusing families: Short term cognitive coercitive group therapy using goal attainment scaling ", dans A.R. Roberts (dir.), Crisis intervention handbook. Assessment, treatment and research, Belmont, CA, Wadsworth.

Kagan, R. et S. Schlosberg (1989). Families in Perpetual Crisis, New York, W.W. Norton et Company.

KINNEY, J., D. HAAPALA et C. BOOTH (1991). Keeping families together: The Homebuilders Model, New York, A. de Gruyter. 
LARIVIĖRE, C. et C. BARBEAU (1999). Rapport final de la recherche effectuée aux Centres jeunesse de Lanaudière, Document inédit, Réseau conseil interdisciplinaire du Québec, Joliette, Québec, Centres jeunesse de Lanaudière.

LeCompte, Y. et Y. LefebVRE (1986). "L'intervention en situation de crise », Santé mentale au Québec, vol. 11, p. 122-142.

LINTON, R. (1989). "Conceptualizing feminism, clarifying social science concepts ", Evaluation and Program Planning, vol. 12, p. 25-29.

MANNING-KENDRICK, J. (1991). "Crisis intervention in child abuse: A family treatment approach », dans A.R. Roberts (dir.), Contemporary perspectives on crisis intervention and prevention, Englewood Cliffs, NJ, Prentice-Hall.

McCown, W.G. et J. JoHNSON (1993). Therapy with treatment resistant families: A consultation-crisis intervention model, New York, Haworth Press.

Miles, M.B. et A.M. Huberman (1994). Qualitative data analysis: An expanded sourcebook ( $2^{\mathrm{e}}$ éd.), Newbury Park, NY, Sage.

MINUCHIN, S. (1974). Families and family therapy, Londres, Tavistock.

Nelson, K.E., M.J. Landsman et W. Deutelbaum (1990). "Three models of family-centered placement prevention services", Child Welfare, vol. 114, p. 1-21.

O'HAGAN, K. (1984). "Family crisis intervention in social services", Journal of Family Therapy, vol. 6, p. 149-181.

PATTON, M.Q. (1986). Utilization-focused Evaluation ( $2^{\mathrm{e}}$ éd.), Newbury Park, NY, Sage.

PATTON, M.Q. (1989). "A context and boundaries for theory-driven approach to validity », Evaluation and Program Planning, vol. 12, p. 375-377.

PATTON, M.Q. (1990). Qualitative Evaluation and Research Methods ( $2^{\mathrm{e}}$ éd.), New York, Sage.

PATTON, M.Q. (1997). Utilization-focused Evaluation (3e éd.), Newbury Park, NY, Sage.

PIEPER, H.M. (1985). "The future of social work research », Social Work Abstracts, vol. $21, \mathrm{n}^{\circ} 4$, p. 3-11.

PITTMAN, F.S. (1986). Turning points : Treating families in transition and crisis, New York, W.W. Norton et Company.

POLKINGHORNE, D. (1988). Narrative knowing and the human sciences, Albany, NY, State University of New York Press. 
Posavac, E.J. et R.G. Carey (1992). Program evaluation: Methods and case studies (2e éd.), Englewood Cliffs, NJ, Prentice-Hall.

PURYEAR, D.A. (1979). Helping people in crisis: A practical familyoriented approach to effective crisis intervention, San Francisco, Jossey-Bass.

REASON, P. (1994). "Three approaches to participative inquiry », dans N.K. Denzin et Y.S. Lincoln (dir.), Handbook of qualitative research, Newbury Park, NY, Sage.

Roberts, A.R. (1990). "An overview of crisis theory and crisis intervention », dans A.R. Roberts (dir.), Crisis intervention handbook. Assessment, treatment and research, Belmont, CA, Wadsworth.

Roberts, A.R. (1991). "Conceptualizing crisis theory and the crisis intervention model », dans A.R. Roberts (dir.), Contemporary perspectives on crisis intervention and prevention, Englewood Cliffs, NJ, Prentice-Hall.

SCHORR, L.B. et D. SCHORR (1988). Within our Reach: Breaking the Cycle of Disadvantage, New York, Anchor Books.

SLIVE, A.B. et N. MCCONKEY-RADETZKI (1989). "The utilization of residential treatment as family crisis intervention », Journal of Child Care, vol. 3, p. 59-68.

SOCIÉTÉ ROYALE DU CANADA (1995). Recherche participative et promotion de la santé, Bilan des recommandations pour le développement de la recherche participative en promotion de la santé au Canada, Institute of Health Promotion Research, The University of British Columbia et le B.C. Consortium for Health Promotion Research.

STEeLE, W. et M. RAIDER (1991). Working with families in crisis : Schoolbased intervention, New York, Guilford.

StRAUSS, A. et J. Corbin (1990). "Basics of qualitative research », Grounded theory procedures and techniques, Newbury Park, NY, Sage.

StRauss, A. et J. CoRBIN (1997). Grounded theory in practice, Newbury Park, NY, Sage.

STRINGER, E.T. (1996). Action research: A handbook for practioners, Newbury Park, NY, Sage.

SUGARMAN, S. et C. MASHESTER (1985). "The family crisis intervention literature: What is meant by "family"? » Journal of Marital and Family Therapy, vol. 2, p. 167-177. 
SugARMAN, S. et C. MASHESTER (1986). "The family crisis intervention literature : What is meant by crisis? "International Journal of Family Psychiatry, vol. 7, p. 359-376.

TANDON, S.D., S.S. AzeLton, J.G. Kelly et D.A. StRICKLAND (1998). "Constructing a tree for community leaders: Contexts and processes in collaborative inquiry ", American Journal of Community Psychology, vol. 26, p. 669-696.

TROCHIM, W.M.K. (1989a). "An introduction to concept mapping for planning and evaluation », Evaluation and program planning, vol. 12, p. 1-16.

TROCHIM, W.M.K. (1989b). "Concept mapping: Soft science or hard art? " Evaluation and Program Planning, vol. 12, p. 87-100.

TROCHIM, W.M.K., J.A. CoOK et R.J. SETZE (1994). "Using concept mapping to develop a conceptual framework of staff's views of a supported employment program for individuals with severe mental illness », Journal of Consulting and Clinical Psychology, vol. 62, $\mathrm{n}^{\circ} 4$, p. $766-775$.

TURNER, B.A. (1983). "The use of grounded theory for the qualitative analysis of organizational behaviour ", Journal of Management Studies, vol. 20, p. 333-348.

UmANA, R., S.J. Gross et M.T. McConvILLE (1980). Crisis in the Family : Three Approaches, New York, Gardner Press.

VINEY, L.L. (1976). "The concept of crisis: A tool for clinical psychologists ", Bulletin of the British Psychological Society, vol. 29, p. 387-395.

WeitZMAN, J. (1985). "Engaging the severely dysfunctional family in treatment : Basic considerations », Family Process, vol. 24, p. 374485.

Wiener, R.L., D. Wiley, T. Huelsman et A. Hilgemann (1994). « Needs assessment. Combining qualitative interviews and concept mapping methodology », Evaluation Review, vol. 18, p. 227-240.

WILSON, H.S. et S.A. HUTCHISON (1991). "Triangulation of qualitative methods: Heideggerian hermeneutics and grounded theory ", Qualitative Health Research, vol. 1, p. 263-276. 